\title{
Late-onset Neutropenia in Patients with Rheumatoid Arthritis after Treatment with Rituximab
}

\author{
Rita Abdulkader, Chethana Dharmapalaiah, Ginny Rose, Lynne M. Shand, Gavin P. Clunie, \\ and Richard A. Watts
}

\begin{abstract}
Objective. Late-onset neutropenia (LON) is an adverse effect of rituximab (RTX) in hematological malignancies, a finding that was recently reported in rheumatoid arthritis (RA). The aim of our study was to estimate its incidence in RA.

Methods. We retrospectively reviewed complete blood (cell) count of patients with RA who received RTX between October 2007 and July 2011 to identify neutropenia $\left(\leq 1.5 \times 10^{9}\right)$ up to 12 months following RTX.

Results. One hundred eight patients received RTX, median age 64 years (range 25-86). A total of 237 cycles were given. Five patients developed LON after a median of 151 days (71-184). Two developed pneumonia.

Conclusion. LON occurs infrequently after RTX, but can present with infection. (First Release March 15 2014; J Rheumatol 2014;41:858-61; doi:10.3899/jrheum.130526)
\end{abstract}

Key Indexing Terms:

RHEUMATOID ARTHRITIS

ADVERSE EFFECTS

\section{BIOLOGICAL THERAPY NEUTROPHILS}

Rituximab (RTX) is a chimeric anti-CD20 monoclonal antibody, licensed for the treatment of severe active rheumatoid arthritis (RA) that is unresponsive to other disease-modifying antirheumatic drugs (DMARD), including at least 1 tumor necrosis factor (TNF)- $\alpha$ inhibitor.

Late-onset neutropenia (LON), occurring 4 weeks following the last administration of RTX with no other identifiable cause, has been recognized as a late adverse effect of RTX therapy for $\beta$-cell lymphomas $1,2,3,4$.

LON has also been reported infrequently in patients treated with RTX for autoimmune conditions, including $\mathrm{RA}^{1,5}$. Tesfa, et al ${ }^{1}$ conducted a retrospective analysis of 209 patients with a variety of autoimmune conditions receiving RTX and identified 11 patients with LON, defined as an

From the Research and Development Department, Department of Rheumatology, The Ipswich Hospital NHS Trust, Ipswich, UK.

$R A W$ and GPC have received support from the Norfolk and Suffolk Comprehensive Local Research Network.

R. Abdulkader, MD, Rheumatology specialist trainee, Rheumatology Department, Norfolk and Norwich University Hospital, Norwich; C. Dharmapalaiah, $M B, B S$, Rheumatology specialist trainee, Rheumatology Department, Basildon and Thurrock University Hospitals NHS Foundation Trust, Basildon; G. Rose, RN, BSc (Hons), Senior research nurse, Research and Development Department, The Ipswich Hospital NHS Trust; L.M. Shand, MB, ChB, Consultant rheumatologist, Rheumatology Department, The Ipswich Hospital NHS Trust; G.P. Clunie, $M B, B S, M D$, Consultant rheumatologist, Cambridge University Hospitals NHS Foundation Trust, Cambridge; R.A. Watts, BM, BCh, DM, Consultant rheumatologist, Rheumatology Department, The Ipswich Hospital NHS Trust.

Address correspondence to Dr. R. Abdulkader, Norfolk and Norwich University Hospital, Colney Lane, Norwich, NR4 7UY, UK.

E-mail: ritaadel@doctors.org.uk

Accepted for publication December 30, 2013. absolute neutrophil count (ANC) of $\leq 1.5 \times 10^{9} / 1$. In their study, LON was more common in patients with granulomatosis with polyangiitis $(23 \%)$ and systemic lupus erythematosus (SLE; 20\%) than in RA (3\%). Seven of the 11 patients were hospitalized with sepsis or febrile neutropenia.

The aim of our study was to assess the incidence of LON and its consequences in a cohort of patients receiving RTX for the routine treatment of RA.

\section{MATERIALS AND METHODS}

The study was conducted in a real-life setting and patients received the conventional standard of care.

Patients. Using our day unit database at the Ipswich Hospital NHS Trust, we identified the patients with RA who attended for RTX infusions between October 2007 (following the introduction of National Institute for Health and Clinical Excellence guidance approving the use of RTX for RA in the UK) and July 2011. We used the hospital pathology results database, clinic letters, and case notes to identify patients who developed neutropenia up to 12 months following the last dose of RTX. LON was defined as an ANC of $\leq 1.5 \times 10^{9} / 1$ occurring 4 weeks or more after the last administration of RTX, for which no other reason was identified ${ }^{1}$. Pretreatment neutropenia cases were excluded. For each patient we recorded demographics, rheumatoid factor, anticitrullinated protein antibodies serology, concurrent DMARD, the number of RTX cycles, and absolute neutrophil count pretreatment, and up to 1 year after the last cycle.

Treatment regimen. Each cycle consisted of 2 doses of RTX, $1 \mathrm{~g}$, intravenously (IV), given 2 weeks apart. Each dose was preceded by $100 \mathrm{mg} \mathrm{IV}$ methylprednisolone. RTX cycles were repeated as needed, but not more than once every 6 months. The frequency of complete blood (cell) count $(\mathrm{CBC})$ was determined by the requirements for the concurrent DMARD. For patients who were not taking a DMARD, CBC was monitored every 3 months, or at the time of clinical review, in accordance with our standard practice at the time. B cell counts and immunoglobulin levels were not routinely checked. 


\section{RESULTS}

During the study, 108 patients ( $72 \%$ female) with a clinical diagnosis of RA were treated with RTX. The demographics of the study group are shown in Table 1 . The total number of RTX cycles given was 237. Median number of cycles received was 2 (range 1-8), mean of 2.2.

Sixty-nine patients $(63.8 \%)$ took a concomitant DMARD throughout the RTX treatment. Six patients took a DMARD for part of the treatment. Thirty-three patients did not take a DMARD because of previous intolerance. The most common DMARD used was methotrexate (45 patients), followed by sulfasalazine (20 patients). Eighteen patients received a combination of DMARD. Other DMARD used included leflunomide, azathioprine, hydroxychloroquine, gold, mycophenolate mofetil, and cyclosporine.

Neutropenia developed in 8 patients. Three cases were excluded: 2 caused by the concomitant DMARD (recent increase in dose, organ failure resulting in toxicity). In the third case, neutropenia was likely related to metastatic malignancy.

In 5 cases neutropenia occurred more than 4 weeks after the last infusion of RTX and no other cause was identified. All 5 patients had seropositive erosive disease. Neutropenia occurred after a median of 151 days (range 71-184), mean of 142 days, following the last infusion. Three patients were also taking DMARD. The DMARD dose had been stable for at least 19 months before the onset of the neutropenic episode with no previous neutropenia attributable to their use. Four patients were treated with oral prednisolone, with at least 1 cycle of RTX up to the neutropenic episode; doses varied between 5 and $15 \mathrm{mg}$.

Prior to starting RTX, patients received a median of 5 (range 4-6) DMARD. Previous DMARD included methotrexate, sulfasalazine, hydroxychloroquine, leflunomide, azathioprine, cyclosporine, gold, and penicillamine. Combination therapy was also previously used in 3 patients.

One TNF- $\alpha$ inhibitor was used in 3 patients, one of whom also received alemtuzumab (anti-CD52) more than 15 years before the neutropenic episode. The other 2 patients took either 2 or 3 TNF- $\alpha$ inhibitors (etanercept, infliximab, and adalimumab) during their disease duration. The characteristics of patients who developed LON are shown in Table 2.

Neutropenic episodes were transient, with a maximum duration of 15 days. There was no associated reduction in red blood cell or platelet count from the baseline. In 2 patients (40\%), neutropenia was complicated by pneumonia. As a result, 1 of those patients received granulocyte colony stimulating factor (G-CSF). The other patient's neutrophil count recovered spontaneously.

The second patient who received G-CSF had 2 episodes of neutropenia. The first episode was asymptomatic (ANC nadir of $\left.1.1 \times 10^{9} / 1\right)$ and lasted 14 days. The second episode was more severe (ANC of 0). It occurred 21 days after the recovery of the neutrophil count and was diagnosed following joint replacement surgery, although this was not complicated by sepsis; prophylactic oral antibiotics and G-CSF were given to avoid complications relating to the surgery. No other cause was identified for either episode and there were no further episodes of neutropenia. Bone marrow aspirate was performed in case 1 . It revealed left shifted granulopoiesis consistent with maturation arrest.

Three patients were re-treated with RTX following the neutropenic episodes, with no recurrence of neutropenia for at least 6 months following the repeat cycle.

\section{DISCUSSION}

Our study describes the occurrence of LON in a single-center cohort of patients with RA treated with RTX in routine clinical practice. Neutropenia occurred in 5 patients (4.6\%). Two patients developed septic complications requiring IV antibiotic, suggesting that there is a significant risk of infection due to LON. Our study supports previous data reporting a lower frequency (3\%) of LON in patients with RA than SLE or antineutrophil cytoplasmic antibody-associated vasculitis (AAV) ${ }^{1}$.

Neutropenia occurring in RA may also be due to Felty's syndrome or large granular lymphocyte leukemia, but these conditions will induce long-lasting neutropenia, not the transient condition that we observed.

Table 1. Characteristics of the study group.

\begin{tabular}{lccc}
\hline Characteristics & All Patients & LON & No LON \\
\hline No. patients & 108 & 5 & 103 \\
Age at first RTX, yrs (range) & $64(25-86)$ & $55(25-63)$ & $65(32-86)$ \\
Ratio female:male & $78: 30$ & $4: 1$ & $74: 29$ \\
Median disease duration at first RTX, yrs (range) & $15(2-41)$ & $18(10-24)$ & $15(2-41)$ \\
Seropositivity & $100(92.5 \%)$ & $5(100 \%)$ & $95(92 \%)$ \\
Erosions & $84(77 \%)$ & $5(100 \%)$ & $79(76 \%)$ \\
Median no. RTX treatments (range) & $2(1-8)$ & $1(1-7)$ & $2(1-8)$ \\
Median no. previous DMARD (range) & $4(2-9)$ & $5(4-6)$ & $4(2-9)$ \\
Median no. previous biologics (range) & $1(0-4)$ & $2(1-3)$ & $1(0-4)$ \\
\hline
\end{tabular}

LON: late-onset neutropenia; RTX: rituximab; DMARD: disease-modifying antirheumatic drugs. 
Table 2. Characteristics of patients who developed late-onset neutropenia.

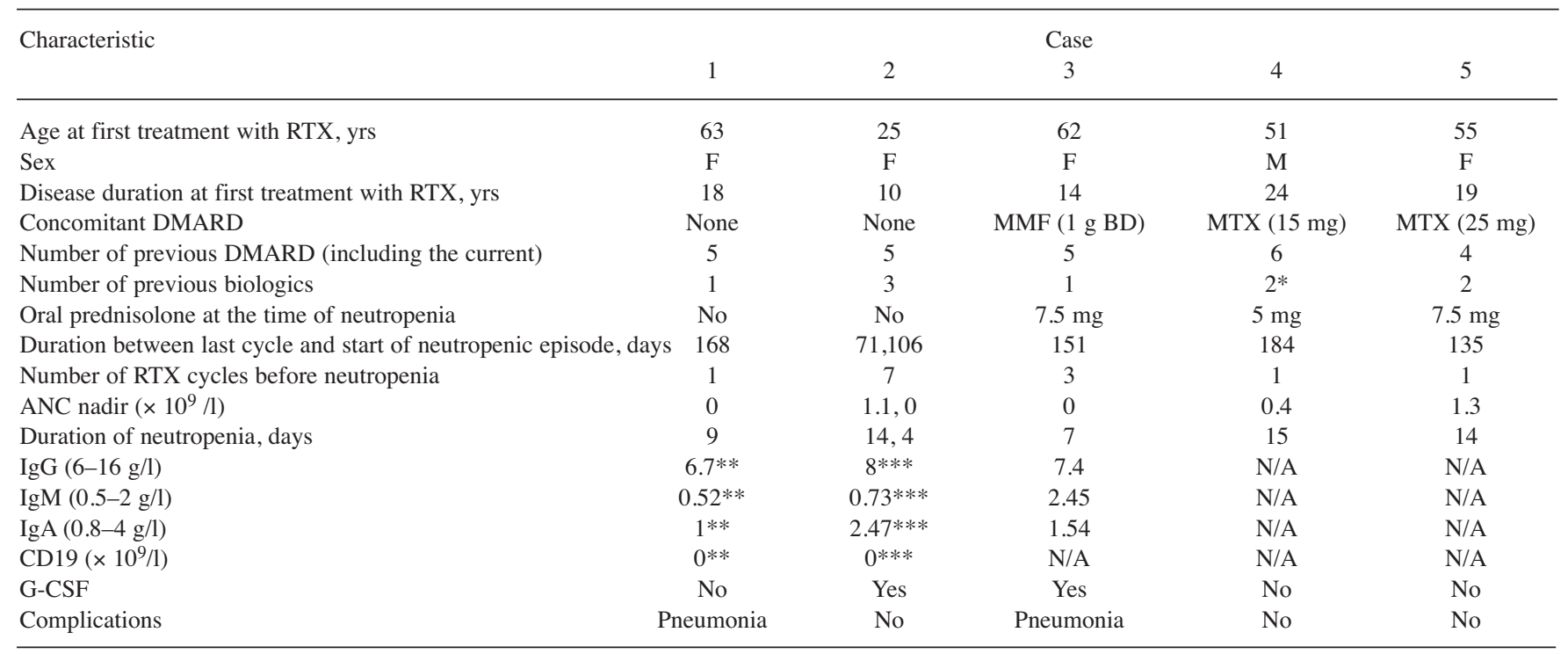

* One TNF- $\alpha$ inhibitor and alemtuzumab. ** Two weeks after the neutropenic episode. ***Five weeks after the neutropenic episode. TNF: tumor necrosis factor; RTX: rituximab; DMARD: disease-modifying antirheumatic drugs; MMF: mycophenolate mofetil; MTX: methotrexate; ANC: absolute neutrophil count; Ig: immunoglobulin; G-CSF: granulocyte colony stimulating factor; N/A: not available.

The mechanisms by which RTX causes LON are not well understood. Maturation arrest at the myelocytic and promyelocytic stages of granulocytes has been observed in bone marrow examination in patients with $\mathrm{LON}^{5}$. Host factors such as polymorphism in the immunoglobulin-G Fc receptor (Fc $\gamma$ RIIIa $158 \mathrm{~V} / \mathrm{F}$ ) were linked to increased risk of LON after RTX treatment for $\beta$ cell lymphoma ${ }^{6,7}$. Direct toxicity has been considered unlikely because of the lack of CD20 on the surface of granulocytes and their precursors ${ }^{2,4}$. Reports of antineutrophil antibodies in patients who developed LON after treatment with RTX suggested an immune-mediated mechanism ${ }^{4}$; however, there has not been enough support for this theory ${ }^{2}$. Another suggested mechanism is through effects of $\beta$ cell recovery on granulocyte homeostasis, resulting in slowing of egression of neutrophils from bone marrow ${ }^{2,3}$. B cell recovery may disrupt bone marrow gradients of stromal cell-derived factor 1 , which may play a role in LON through its effects on myelopoiesis and bone marrow retention of neutrophils ${ }^{3,8}$.

A possible explanation for the higher frequency of LON seen in AAV and SLE is that these patients tend to be more heavily immunosuppressed than patients with $\mathrm{RA}^{9}$. In our study, patients who developed LON had a longstanding severe disease with previous multiple treatments, including at least 1 TNF- $\alpha$ inhibitor. Whether with modern treatment protocols and earlier use of RTX the frequency of LON will be lower still needs to be investigated.

The major weakness of our study is that we did not have routine $\mathrm{CBC}$ for all patients. We measured only the neutrophil count, as required for DMARD monitoring or when patients attended clinic for review. It is possible, accordingly, that we underestimated the incidence of LON, considering its transient nature. Uncomplicated neutropenic episodes might have been missed between routine tests. A key issue raised by our study and the work by Tesfa, et $a l^{1}$ is whether the neutrophil count should be routinely monitored and if so, how frequently.

We suggest that rheumatologists should be aware of late onset neutropenia and the increased risk of infectious complications. It is also important to educate patients to seek medical advice and have their neutrophil count checked should they develop an infection.

\section{ACKNOWLEDGMENT}

The authors thank Pamela Bradely and the audit department at the Ipswich hospital NHS trust.

\section{REFERENCES}

1. Tesfa D, Ajeganova S, Hägglund H, Sander B, Fadeel B, Hafström $\mathrm{I}$, et al. Late-onset neutropenia following rituximab therapy in rheumatic diseases: association with B lymphocyte depletion and infections. Arthritis Rheum 2011;63:2209-14.

2. Wolach O, Bairey O, Lahav M. Late-onset neutropenia after rituximab treatment: case series and comprehensive review of the literature. Medicine 2010;89:308-18.

3. Fukuno K, Tsurumi H, Ando N, Kanemura N, Goto H, Tanabashi S, et al. Late-onset neutropenia in patients treated with rituximab for non-Hodgkin's lymphoma. Int J Hematol 2006;84:242-7.

4. Voog E, Morschhauser F, Solal-Céligny P. Neutropenia in patients treated with rituximab. N Engl J Med 2003;348:2691-4.

5. Tesfa D, Gelius T, Sander B, Kimby E, Fadeel B, Palmblad J, et al. Late-onset neutropenia associated with rituximab therapy: evidence for a maturation arrest at the (pro)myelocyte stage of 
granulopoiesis. Med Oncol 2008;25:374-9.

6. Weng WK, Negrin RS, Lavori P, Horning SJ. Immunoglobulin G Fc receptor FcgammaRIIIa 158 V/F polymorphism correlates with rituximab-induced neutropenia after autologous transplantation in patients with non-Hodgkin's lymphoma. J Clin Oncol 2010; 28:279-84.

7. Li SC, Chen YC, Evens AM, Lee CC, Liao HF, Yu CC, et al. Rituximab-induced late-onset neutropenia in newly diagnosed B-cell lymphoma correlates with Fc receptor Fc $\gamma$ RIIIa 158(V/F) polymorphism. Am J Hematol 2010;85:810-2.
8. Dunleavy K, Hakim F, Kim HK, Janik JE, Grant N, Nakayama T, et al. B-cell recovery following rituximab-based therapy is associated with perturbations in stromal derived factor- 1 and granulocyte homeostasis. Blood 2005;106:795-802.

9. Besada E. Alternative explanations for development of late-onset neutropenia in rituximab-treated rheumatic disease patients: comment on the article by Tesfa et al [letter]. Arthritis Rheum 2012;64:596-7. 